\title{
Capillary Action-Supported Contactless Atmospheric Pressure Ionization for the Combined Sampling and Mass Spectrometric Analysis of Biomolecules
}

\author{
Cheng-Huan Hsieh, Chia-Hsien Chang, Pawel L. Urban, and Yu-Chie Chen* \\ Department of Applied Chemistry, National Chiao Tung University, Hsinchu, Taiwan \\ Supporting Information
}

ABSTRACT: It is proposed that a short tapered capillary can be utilized as a nanoliter-volume sampling tool and sample emitter for generation of gas-phase ions in front of the mass spectrometer, without the need for using an additional electric power supply, a gas supply, or a syringe pump. A wide range of molecules can be analyzed in pure solutions and complex matrixes (cell extract, urine, and plant tissue) with no or minimum sample preparation. Singly and multiply charged ions can be detected in either positive or negative-ion mode. Because of the nanoliter-volume sampling and low spectral background, the mass detection limit for bradykinin is in the low attomole range. Other advantages include simplicity, disposability, and low cost. The putative mechanism of the ion formation in this capillary-action supported contactless spray emitter is discussed.

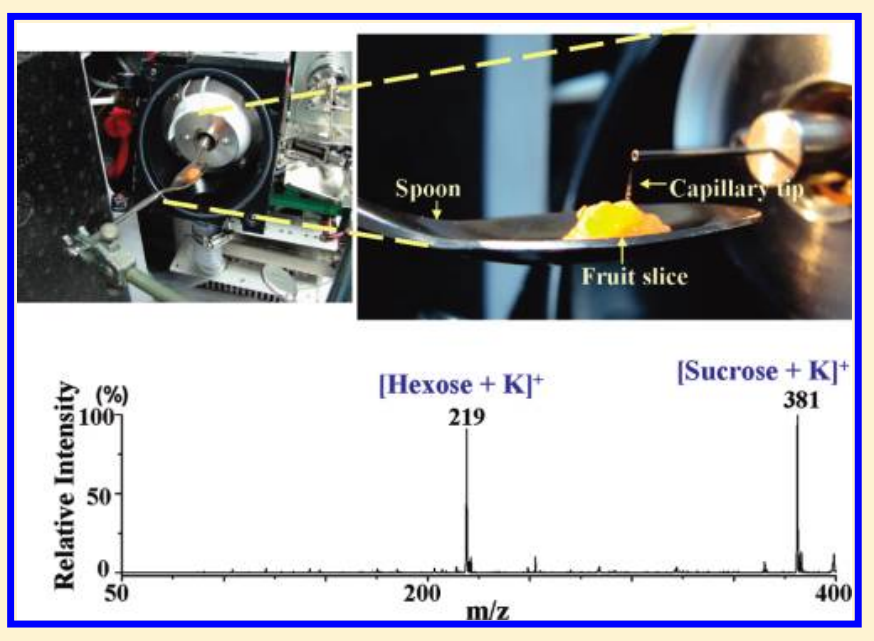

The invention of electrospray ionization (ESI) provided a versatile tool for mass spectrometric (MS) measurements of biomolecules and enabled ground-breaking discoveries in bioscience. $^{1-3}$ Formation of gas-phase ions in electrospray ionization mass spectrometry (ESI-MS) usually relies on applying an external electric potential to the ESI emitter. ${ }^{4,5}$ However, connecting an electric potential to the spray emitter is not always required to generate gas-phase ions. ${ }^{6-8}$ For example, in the miniaturized version of ESI (nanoESI), application of an electric potential to the upstream part of the flow line can be sufficient to create an electrospray at the outlet of the capillary channel. Fused-droplet and extractive electrospray ionization, ${ }^{9-12}$ as well as sonic spray ionization, ${ }^{13-15}$ Venturi easy ambient sonic-spray ionization (V-EASI) ${ }^{16}$ or ultrasonication-assisted spray ionization (UASI) ${ }^{17,18}$ methods have successfully been used to generate gas-phase ions without an electric potential applied to the sample emitter.

This leads to a hypothesis that the production of fine droplets and subsequent desolvation, not the application of an electric potential to the emitter, are required to generate gas-phase ions in a continuous manner. To verify this assumption, here we demonstrate a simple continuous-flow ion source that neither requires connection to an electric power supply to the sample emitter nor uses any other instrumental aids, such as gas supply, syringe pump, laser, or ultrasonic transducer, to facilitate transfer of the sample from the liquid into the gas phase. For the convenience of description, we refer to it as "contactless atmospheric pressure ionization” (or "contactless API”).

The contactless API setup consists of only few easily available and inexpensive components (Figure 1): A short capillary is used to drive liquid samples from a microscale reservoir toward the outlet, and fine droplets are expected to be formed at the tapered tip positioned in the proximity of the MS orifice. Because of the capillary action, continuous flow of the sample toward the tapered end of the capillary is generated, followed by immediate transfer of the liquid-phase analytes into the gas phase.

Figure 2a displays the contactless API mass spectrum of a test sample, bradykinin $\left(10^{-6} \mathrm{M}\right.$, in water/acetonitrile $\left.1: 1, \mathrm{v} / \mathrm{v}\right)$ : the prominent signal at $\mathrm{m} / z 531$ corresponds to doubly charged bradykinin ions. The limit of detection for bradykinin was $\sim 1$ $\mathrm{nM}$ (based on the $\mathrm{S} / \mathrm{N}=6$ criterion, see the inset in Figure 2a). Taking into account the estimated flow rate of the sample in the silica capillary $\left(\sim 3 \mathrm{~nL} \mathrm{~min}^{-1}\right)$, and the data acquisition time of 1 $\mathrm{min}$, this figure corresponds to the analyte amount of $\sim 5$ amol. Beside the nanoliter-volume sampling, one other reason for the relatively good mass sensitivity of the method is the low spectral noise. This is in line with a previous observation that eliminating an external electric potential on the sample outlet in the

Received: February 24, 2011

Accepted: March 25, 2011

Published: March 29, 2011 


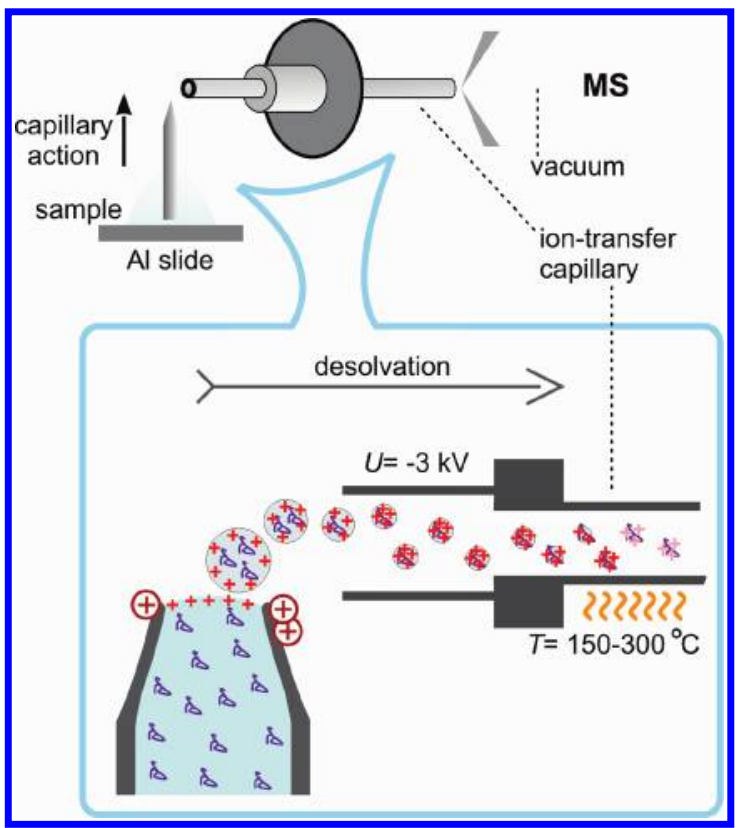

Figure 1. Schematic representation of the contactless atmospheric pressure ionization mass spectrometry, with sample delivery enabled by capillary action. A short tapered silica capillary (length, $1 \mathrm{~cm}$; base o.d., $\sim 363 \mu \mathrm{m}$ (or $\sim 323 \mu \mathrm{m}$ without polyimide); tip o.d., $\sim 10 \mu \mathrm{m}$ ) was positioned vertically above an electrically isolated aluminum slide, with the outlet end placed orthogonal to the inlet of a metal capillary attached to the orifice of an ion trap mass spectrometer. The distance between the outlet of the silica capillary and the inlet of the metal capillary, attached to the MS orifice, was $\sim 1 \mathrm{~mm}$. Before the measurements, the silica capillary was filled with a makeup solution (deionized water/acetonitrile $(1: 1, \mathrm{v} / \mathrm{v}))$ by means of capillary action. The inlet end of the silica capillary was then dipped into a droplet of a sample $(\sim 10 \mu \mathrm{L})$ put onto the surface of the aluminum slide. The inset provides an illustration of the hypothetical mechanism of contactless API.

V-EASI ${ }^{16}$ and UASI ${ }^{17,18}$ could help to reduce the spectral noise due to the presence of solvent ions formed during electrochemical reduction/oxidation. Moreover, the time required for the analysis of samples containing analytes in the nanomolar range was $1 \mathrm{~min}$, resulting in the consumption of just a few nanoliters. However, because of the high $\mathrm{S} / \mathrm{N}$ ratios observed for higher concentrations of analytes (Figure 2), the data acquisition time could still be decreased. After measurement, the microlitervolume sample droplet deposited on the aluminum slide can readily be recycled for further experiments. This is opposed to the ionization devices which incorporate syringes and long tubing with a considerable dead volume, which restrict the possibilities of contamination-free recycling of low-volume $(\leq 10 \mu \mathrm{L})$ samples.

By means of contactless API-MS, it is possible to analyze a wide range of small molecule compounds. Signals of protonated amino acids, including histidine, phenylalanine, and arginine at $m / z 156,166$, and 175 , respectively, could readily be observed in the contactless API mass spectra (Figure 2b). Contactless APIMS is also applicable to the analysis of other peptides and proteins. Figure $2 c-e$ presents the contactless API mass spectra of melittin, cytochrome $\mathrm{C}$, and myoglobin, respectively, which are dominated by the peaks of multiply charged analyte ions. Therefore, similar to ESI, inexpensive MS instruments with limited mass range may be used in conjunction with contactless API emitters to analyze large biomolecules. The interface can

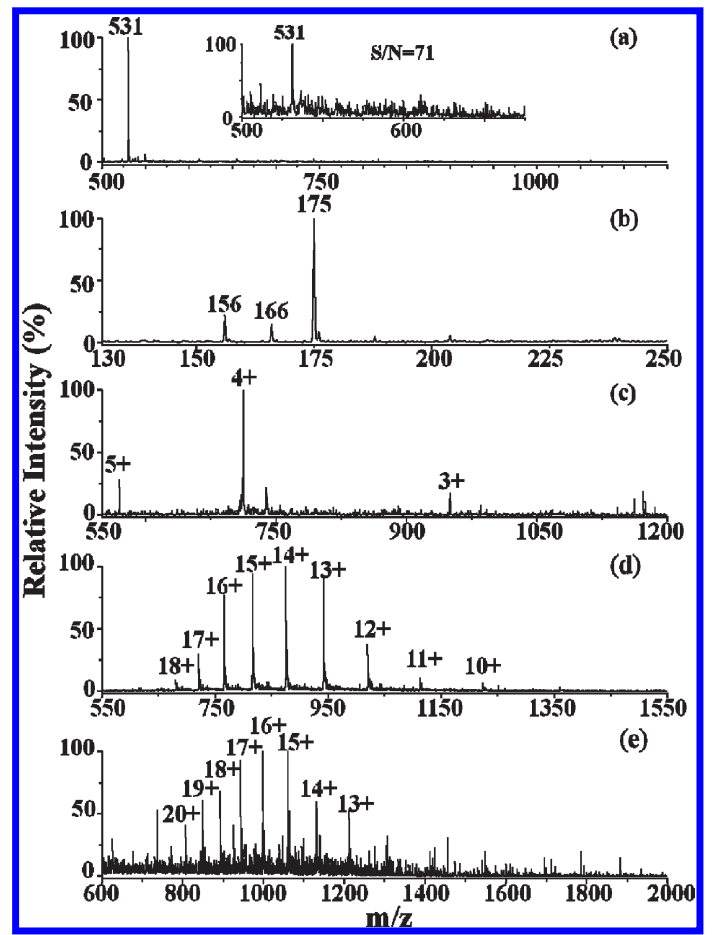

Figure 2. Contactless API mass spectra of (a) bradykinin $\left(10^{-6} \mathrm{M}\right.$; inset, $\left.10^{-8} \mathrm{M}\right)$, (b) a mixture $\left(10^{-5} \mathrm{M}\right)$ of histidine $(\mathrm{m} / \mathrm{z} 156)$, phenylalanine $(\mathrm{m} / z 166)$, and arginine $(\mathrm{m} / z 175),(\mathrm{c})$ melittin $\left(10^{-5}\right.$ $\mathrm{M})$, (d) cytochrome C $\left(10^{-4} \mathrm{M}\right)$, and (e) myoglobin $\left(10^{-4} \mathrm{M}\right)$. The peak at $m / z 531$ in part a corresponds to the doubly charged form of bradykinin. Temperature of the ion-transfer capillary/dry gas was normally set to $150{ }^{\circ} \mathrm{C}\left(300^{\circ} \mathrm{C}\right.$ for the inset in part a).

operate as long as the sample solution is present at the inlet of the silica capillary.

Further on, contactless API-MS is shown to be suitable for the analysis of real samples without or with minimum sample preparation. A slice of muskmelon punched with a contactless API capillary $(\sim 1 \mathrm{~cm}$, cf. inset in Figure $3 \mathrm{a})$ was directly analyzed by contactless API-MS. In this experiment, the contactless API capillary also played the role of a micrometer-scale sampling probe. That is, on the basis of the capillary action, the juice contained in muskmelon was automatically drawn toward the tapered capillary end for instantaneous contactless API-MS analysis. Signals of the potassium adducts of hexose (e.g., glucose and fructose) and sucrose appear at $\mathrm{m} / \mathrm{z} 219$ and 381, respectively (Figure 3a): this is presumably due to the high abundance of potassium ions in plant tissue. This result indicates that the contactless API-MS is suitable for analysis of watery samples without any sample pretreatment. Here, capillary action facilitates in situ sampling of nanoliter-volumes of fluid required to obtain high-quality mass spectra. Therefore, extraction of the juice from the fruit sample to fill up a syringe as well as purification and dilution are avoided. Contactless API-MS can also be used for analysis of biological samples such as cell lysates. Figure $3 \mathrm{~b}$ displays the negative-ion mode contactless API mass spectrum of the HeLa cell extract with many interesting features, primarily corresponding to lipids (in particular, phospholipids); for instance, the peaks at $m / z 883.6,885.6$, and 887.6 are identified by tandem mass spectrometry (MS/MS) and database searches as phosphatidylinositols (Figure S1 in the Supporting Information). Figure $3 \mathrm{c}$ presents the contactless API mass 


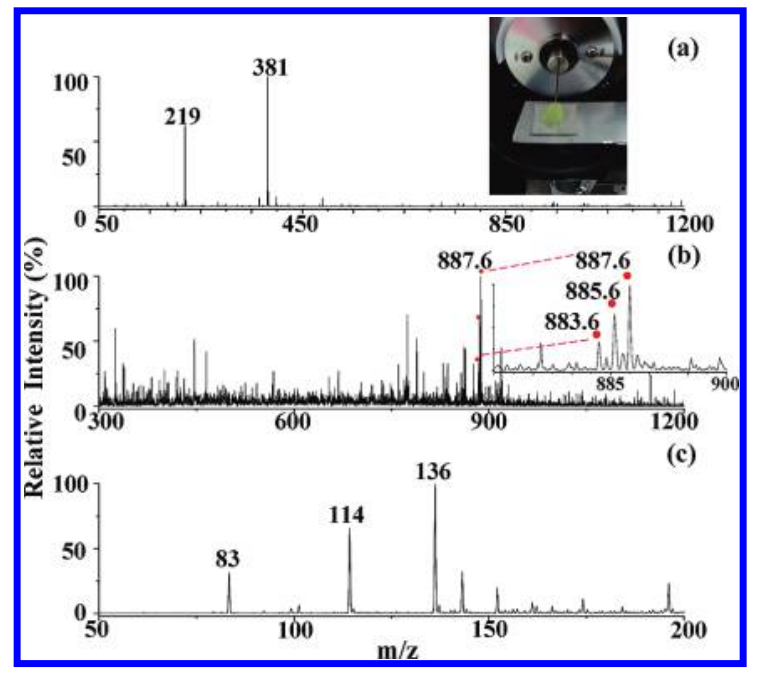

Figure 3. Contactless API mass spectra obtained from (a) a slice of muskmelon, (b) a HeLa-cell extract, and (c) a urine sample diluted 10× with solvent (deionized water/acetonitrile $1: 1, \mathrm{v} / \mathrm{v}$ ). The spectra in parts $a$ and $c$ were obtained in the positive-ion mode, while part b was obtained in the negative-ion mode. The temperature of the ion-transfer capillary/ dry gas was set to $300^{\circ} \mathrm{C}$.

spectrum of a urine sample diluted $10 \times$ in the mixture of acetonitrile and water $(1: 1(\mathrm{v} / \mathrm{v}))$. In the mass range 50-200 $\mathrm{Da}$, several peaks have a particularly high $\mathrm{S} / \mathrm{N}$ ratio: the peak at $\mathrm{m} / z 83$ corresponds to the sodium adduct of urea, while the peaks at $m / z 114$ and 136 correspond to the protonated and sodiated forms of creatinine, respectively. According to literature, the concentration of sugars such as sucrose or glucose in muskmelon can be up to $25 \mathrm{mg} \mathrm{g}^{-1} \mathrm{FW},{ }^{19}$ while the average concentrations of urea and creatinine in urine are in the order of 10 and $1 \mathrm{mg} \mathrm{mL}^{-1}$, respectively. ${ }^{20}$ This is in line with the observation of high signals corresponding to those metabolites in contactless API mass spectra. Overall, these results show that the contactless API-MS method copes with analysis of complex samples, such as unpurified cell extracts and urine, and it can readily be used for analysis of metabolites in volume-limited samples.

Successful operation of this simplistic sampling/ionization setup raises questions regarding the mechanism involved in ion formation. Although the contactless API emitter is not physically connected to any power supply, the electric field present near the MS orifice (due to application of an electric potential to the metal capillary) can cause polarization of the dielectric materials and charge separation near the outlet of the silica capillary (Figure 1). This can occur because the outlet end of the emitter is closer to the metal capillary (connected to high voltage) than the lower end, and the magnitude of electric field emanating from the metal capillary at the MS orifice is inversely proportional to the square of the distance. The anticipated charge separation can subsequently cause accumulation of positive charges at the meniscus, as long as a negative potential is applied to the metal capillary at the MS orifice, when the setup is operated in positive-ion mode (Figure 1).

In order to verify the influence of the tip geometry, a tapered capillary (Figure 2a and Figure S2a in the Supporting Information) as well as two untapered capillaries (a capillary without polyimide coating (o.d., $\sim 323 \mu \mathrm{m}$; i.d., $50 \mu \mathrm{m}$; cf. inset in Figure S2b in the Supporting Information) and a capillary with polyimide coating (o.d., $\sim 363 \mu \mathrm{m}$; i.d, $50 \mu \mathrm{m}$; cf. inset in Figure
S2c in the Supporting Information)) were tested as contactless API emitters. Unlike in the reference spectrum depicted in Figure S2a in the Supporting Information (same as Figure 2a), using these two capillaries as contactless API emitters, we were unable to observe consistent signals corresponding to bradykinin ions. When the capillary is used without polyimide coating, a lowintensity peak $(\mathrm{m} / z \mathrm{1061})$, corresponding to singly charged species, could only be recorded (Figure S2b in the Supporting Information) for a higher concentration of bradykinin $\left(10^{-5} \mathrm{M}\right)$. When using the capillary with polyimide coating as the contactless API emitter, we were unable to obtain any MS signals for the analytes present in the sample (Figure S2c in the Supporting Information). In this experiment, all the three tips were exposed to an electric field of similar magnitude. However, the surface area of the upper part of the tapered capillary tip is smaller than the surface area of the untapered tip (or the tip with polyimide coating). Therefore, local charge density (after charge separation in heterogeneous electric field) will be higher at the tapered tip than at the untapered tip. Higher charge density at the tip should favor detachment of microdroplets from the meniscus, which can further be desolvated and release gas-phase ions. However, apart from the electric field present near the metal capillary at the MS orifice, also hydrodynamic flow of the liquid sample in silica capillary, as well as flow of dry gas, may partly contribute to detachment of microdroplets from the meniscus. In a video sequence taken during operation of the contactless API emitter (Supporting Information, Video A), it is easy to observe the flow of liquid out of the capillary tip. However, because of the limited spatial and temporal resolution of the imaging system, it is not possible to observe individual droplets detaching from the meniscus at the capillary tip.

Although the process of droplet formation in contactless API is not exactly the same as that described for ESI (occurrence of Taylor cone, jet, and electrospray, while high voltage is supplied to the emitter), ${ }^{21}$ desolvation and ion release from the droplets occurs in a similar way as in normal ESI. Furthermore, even if, unlike in ESI, no external voltage is applied to the emitter, the electrostatic charges accumulated at the tip of the silica capillary help to overcome the surface tension of the liquid and generate a stream of charged droplets (Figure 1). If sufficiently small droplets are formed in this process, they can be easily desolvated during their migration in front of the MS orifice and, further on, inside the heated transfer capillary (Figure 1).

If the liquid aerosols are introduced to the heated transfer capillary in the mass spectrometer, its temperature (as well as the temperature of dry gas) should influence the desolvation process and thereby affect ion yield. To verify this assumption, we varied the temperature of the ion-transfer capillary/dry gas in the mass spectrometer and examined intensities of the MS signals. Figure S3 in the Supporting Information displays the contactless API mass spectra of melittin $\left(10^{-6} \mathrm{M}\right)$ analyzed at various temperatures of the ion-transfer capillary/dry gas (i.e., 150, 200, 250, and $300{ }^{\circ} \mathrm{C}$ ): clearly, the intensities of multiply charged ions of melittin increased with increasing temperature. An increased evaporation of the solvent at higher temperatures increases the abundance of ions entering the MS analyzer, while nondesolvated droplets are most probably lost by hitting surfaces within the early vacuum stage or outside the orifice of the mass spectrometer. Apart from the temperature, similar to ESI, addition of an acid (e.g., acetic acid) can decrease surface tension of the sample solution and therefore facilitate formation of fine 
droplets, which are more prone to desolvation. This is especially relevant when analyzing large biomolecules, such as proteins.

In conclusion, we propose that a short tapered silica capillary can readily be utilized as a nanoliter-volume sampling tool and sample emitter for generation of gas-phase ions at atmospheric pressure without the need for using an additional electric power supply, gas supply, or syringe pump, as long as high voltage is applied to the MS orifice. Similar to ESI-MS, singly and multiply charged ions can be detected in contactless API-MS, operated in either positive or negative-ion mode. Spectral noise is kept at a low level due to the decreased contribution of redox processes. Other advantages include simplicity, disposability, low cost, wide detectable mass range, and low detection limits (nanomolar concentrations or attomole amounts). Contactless API enables analysis of liquid samples in a continuous manner; therefore, in perspective, it is imaginable to combine it with other continuousflow techniques, such as nanoliter flow or capillary-action liquid chromatography, flow injection analysis, and microfluidic devices. In addition, it may be useful to conduct online in situ purification of matrix-rich samples using capillaries with coated walls or filled with a suitable stationary phase to decrease ion suppression and further increase sensitivity. Because of its simplicity and no requirement for external gas or voltage supply, it might also be interesting to apply the contactless API emitters together with portable mass spectrometers to facilitate measurements outside laboratory.

\section{ASSOCIATED CONTENT}

S Supporting Information. Additional experimental details and results/figures (pdf file) and Video A. This material is available free of charge via the Internet at http://pubs.acs.org.

\section{AUTHOR INFORMATION}

\section{Corresponding Author}

*Phone: +886-3-5131527. Fax: +886-3-5723764. E-mail: yuchie@mail.nctu.edu.tw.

\section{ACKNOWLEDGMENT}

We thank the National Science Council of Taiwan for the financial support of this work. We also thank Mr. Po-Han Chan for providing the HeLa cells.

\section{REFERENCES}

(1) Whitehouse, C. M.; Dreyer, R. N.; Yamashita, M.; Fenn, J. B. Anal. Chem. 1985, 57, 675-679.

(2) Fenn, J. B.; Mann, M.; Meng, C. K.; Wong, S. F.; Whitehouse, C. M. Science 1989, 246, 64-71.

(3) Nguyen, S.; Fenn, J. B. Proc. Natl. Acad. Sci. U.S.A. 2007, 104, 1111-1117.

(4) Yamashita, M.; Fenn, J. B. I. Phvs. Chem. 1984, 88, 4451-4459.

(5) Banks, J. F.; Shen, S.; Whitehouse, C. M.; Fenn, J. B. Anal. Chem. 1994, 66, 406-414.

(6) Mazereeuw, M.; Hofte, A. J. P.; Tjaden, U. R.; van der Greef, J. Rapid Commun. Mass Spectrom. 1997, 11, 981-986.

(7) Vrouwe, E. X.; Gysler, J.; Tjaden, U. R.; van der Greef, J. Rapid Commun. Mass Spectrom. 2000, 14, 1682-1688.

(8) Wu, Y.-T.; Chen, Y.-C. Anal. Chem. 2005, 77, 2071-2077.

(9) Shiea, J.; Chang, D.-Y.; Lin, C.-H.; Jiang, S.-J. Anal. Chem. 2001, 73, 4983-4987.
(10) Chang, D.-Y.; Lee, C.-C.; Shiea, J. Anal. Chem. 2002, $74,2465-2469$.

(11) Chen, H.; Venter, A.; Cooks, R. G. Chem. Commun. 2006, 2042-2044.

(12) Chen, H. W.; Zenobi, R. Nat. Protoc. 2008, 3, 1467-1475.

(13) Hirabayashi, A.; Sakairi, M.; Koizumi, H. Anal. Chem. 1994, 66, 4557-4559.

(14) Hirabayashi, A.; Sakairi, M.; Koizumi, H. Anal. Chem. 1995, $67,2878-2882$.

(15) Dams, R.; Benijts, T.; Günther, W.; Lambert, W.; De Leenheer, A. Anal. Chem. 2002, 74, 3206-3212.

(16) Santos, V. G.; Regiani, T.; Dias, F. F. G.; Romão, W.; Jara, J. L. P.; Klitzke, C. F.; Coelho, F. B.; Eberlin, M. N. Anal. Chem. 2011, 83, 1375-1380.

(17) Chen, T.-Y.; Lin, J.-Y.; Chen, J.-Y.; Chen., Y.-C. J. Am. Soc. Mass Spectrom. 2010, 21, 1547-1553.

(18) Chen, T.-Y.; Chao, C.-S.; Mong, K. K. T.; Chen, Y.-C. Chem. Commun. 2010, 46, 8347-8349.

(19) Lingle, S. E.; Dunlap, J. R. Plant Phvsiol. 1987, 84, 386-389.

(20) http://en.wikipedia.org/wiki/Urine (accessed on March 16, 2011).

(21) Kebarle, P.; Verkerk, U. H. Mass Spectrom. Rev. 2009, $28,898-917$. 